\title{
Development of Filipino Phonetically-balanced Words and phoneme-level Hmms
}

\author{
Arnel C. Farjardo ${ }^{1}$, Yoon-Joong Kim ${ }^{2}$ \\ Professor, Department of Computer Science, La Consolacion College, Manila, Philippines ${ }^{1}$ \\ Professor, Department of Computer Engineering, Hanbat National University, Daejeon, Korea ${ }^{2}$
}

\begin{abstract}
In this paper, we developed two sets of phonetically balanced word (PBW) lists in Filipino and two sets of phoneme-level HMMs (Hidden Markov Model). Two PBW lists were based on textbooks used in public school in Philippines and used to develop speech corpus with the fifty speakers of 25 males and 25 females. In a 2-syllable word list (PBW2), an average accuracy rate of $88.95 \%$ for speaker dependent and $82.57 \%$ for speaker independent test were achieved. For 3-syllable word list (PBW3), the recognizer achieved an accuracy rate of $90.28 \%$ for speaker dependent and $83.30 \%$ for speaker independent test.
\end{abstract}

Keywords: Filipino phonetically balanced words, Filipino word corpus, Hidden Markov Model, Automatic Speech Recognition

\section{INTRODUCTION}

Speech is one of the most effective means of human However, no one of these previous researches used a communication that is acquired as a skill through phonetically balanced set of words for the development of interaction within an environment. In the past decades, its speech corpus. Thus, the researchers' objectives are to computer scientists as well as linguists have been studying (1) present the development of Filipino PBW and (2) test effective means of recognizing speech through automated the recognition accuracy of the developed Filipino PBW machines. An Automatic Speech Recognition (ASR) using HMM. This paper will provide some information of converts the speech signals into words. The recognized Filipino language, development of phonetically balancedwords can be the final output or it can be an input for a word lists and the recognition accuracy test for the natural language processing. In the recent years, Filipino phonetically balanced speech.

researchers aimed to provide an accurate speech recognizer [1] [2]. However, no one provides an efficient solution for the Filipino Language.The Hidden Markov Model (HMM) is a doubly stochastic process with one that is not directly observable [3]. This hidden process can be observed only through another set of stochastic process that can produce the observation sequence.

HMMs are so far the widely used acoustic model for speech recognition [4]. This model is used from previous studies relating to an Automatic Speech recognizer for the Filipino Language [1] [2]. In 2003, an ASR for Filipino phonemes was developed [1]. This study reported to have achieved recognition accuracy of $85.5 \%$. However, this recognizer was used to recognize phoneme utterances of the Filipino alphabets using discrete HMM limiting itself for small vocabulary speech corpus.A Filipino Speech Corpus was developed by Guevara R., et. al (2002) to be used for continuous Speech recognition. Dela Roca G., et.al (2003) tested the developed Filipino corpus to recognize continuous speech and achieve $32 \%$ recognition accuracy. In an attempt to increase this accuracy, a study in 2010 was held where an Indonesian speech corpus was used for the recognizer as training sets to recognize Filipino utterances [5]. The Indonesian speech corpus contains 80 hours of recording compared to the developed Filipino speech corpus in 2003 that contains 4 hours of recording. This cross-lingual approach achieved $79.50 \%$ recognition accuracy.

\section{THE FILIPINO LANGUAGE}

Filipino is the language used largely in the Philippines with 22 million native speakers [6].Between the 1930s and mid-1970's, a system of syllabication for the alphabet called abakada was developed by Lope K. Santos to represent the native sounds [7]:

abaka da e ga ha I la ma nanga o

parasa ta u waya

This represents the Filipino alphabet consisting of 5 (a, e, i, o, u) vowels and 15 (b, k, d, g, h, l, m, n, ng, p, r, s, t, w, y) consonants. The Filipino alphabet, though in a sense, considered as phonetic, does not reflect exactly the correct sound in written form [8]. There are words present in the Filipino Language that are spelled the same but are pronounced with slight differences, which produce difference in meaning.

\section{bata /b:a - ta/ - "a child"}

bata /ba - ta/ - "to bear or endure"

The word bata with the phonetic representation of /b:a ta/ denotes a long sound which is produced by a short pause after the affected syllable, while the other phonetic representation /ba-ta/ is produced continuously without breaks.Thus, the Filipino phonemes can be broken down to the following phones:

Vowels :

/a/ /e/ /i/ /o/ /u/ 
Consonants :

$/ \mathrm{b} / / \mathrm{k} / / \mathrm{d} / / \mathrm{g} / / \mathrm{h} / / \mathrm{l} / / \mathrm{m} / / \mathrm{n} / / \mathrm{g} / / \mathrm{p} / / \mathrm{r} / / \mathrm{s} / / \mathrm{t} / / \mathrm{w} / / \mathrm{y} /$

Table 1: The Filipino Vowel System

\begin{tabular}{|c|c|c|c|}
\hline & front & central & back \\
\hline upper & $/ \mathrm{i} /$ & & $/ \mathrm{u} /$ \\
\hline middle & $/ \mathrm{e} /$ & & $/ \mathrm{o} /$ \\
\hline lower & & $/ \mathrm{a} /$ & \\
\hline
\end{tabular}

The Filipino vowel phonemes can be described as /a/ low central unrounded, /e/ mid front unrounded, /i/ upper front unrounded, /o/ mid back rounded, and /u/ upper back rounded. According to tongue height, we have two front vowel phonemes /i e/, and two back vowel phoneme /o u/, and one central vowel phoneme $/ \mathrm{a} /$.

Table 2: The Filipino Consonant System

\begin{tabular}{|c|c|c|c|c|c|c|}
\hline & labial & dental & alveolar & palatal & velar & glottal \\
\hline \multirow{2}{*}{ stops } & voiced & \multirow{2}{*}{$/ \mathrm{p} /$} & $/ \mathrm{t} /$ & & $/ \mathrm{k} /$ & \\
\cline { 2 - 7 } & voiceless & & & & \\
\hline nasals & voiced & $/ \mathrm{b} /$ & $/ \mathrm{d} /$ & & $/ \mathrm{g} /$ & \\
\hline fricatives & voiceless & & $/ \mathrm{n} /$ & & $/ \mathrm{\eta} /$ & \\
\hline affricatives & voiceless & & & $/ \mathrm{s} /$ & & $/ \mathrm{h} /$ \\
\hline lateral & voiced & & & $/ \mathrm{l} /$ & & \\
\hline flap & voiced & & & $/ \mathrm{r} /$ & & \\
\hline glide & voiced & $/ \mathrm{w} /$ & & & $/ \mathrm{y} /$ & \\
\hline
\end{tabular}

The Filipino consonants are produced through the help of the lips (labial), teeth (dental), alveolar ridge (alveolar), palate (palatal), velum (velar), and glottis (glottal).

\section{III.PHONETICALLY BALANCED WORDS}

In the development of an automatic speaker-independent continuous Filipino speech recognizer, a set of properly selected word list is required for the development of a recorded speech corpus. The set of words must be phonetically balanced in nature, and must contain all phonemes present that are characterized by the Filipino language. In the construction of large-vocabulary word recognition, a set of recording must be obtained from a spoken corpus gained from a written corpora or a phonetically balanced word list. A Filipino Speech corpus was developed by Guevara, et. al [2] that includes both an open-ended and close-ended spoken words. This methodology in training data is not phonetically balanced. A phonetically balanced speech text used for English, German, Swedish, Danish, Hebrew, Italian, Finish, French, and Portuguese often taken into the following criteria [9][10]: Syllable structure, equal phonetic structure, phonetic balance, equal average difficulty and equal range of difficulty, common words, and speaker intelligibility.

In [13], a total set of 257 words are produced for the 2syllable word list, and 212 words for the 3-syllable word list. These words are then used for the recording of speech corpora that are used for testing via word recognition. All paragraphs must be indented. All paragraphs must be justified, i.e. both left-justified and right-justified.

\section{A. Development of Phonetically Balanced Word Lists}

The Filipino phonetically balanced words were evaluated from 16 articles found from a Filipino based textbook written for senior public school students, "Bagwis". This textbook is approved by the Department of Education and Sports Commission of the Philippine Government, thus be considered reliable with a minimal chance of error. All the articles extracted from the textbook are written in Filipino, which consists of a total of 9768 words

Table 3: List of Articles and its corresponding word count from the "Bagwis IV"

\begin{tabular}{|l|l|}
\hline Title & Words \\
\hline AngGilingangBato & 2905 \\
\hline Sa YapakngPambansangbayanisa Heidelberg & 1154 \\
\hline AngWika Ng Pilipino At Ang Banta Ng Globalisasyon & 1150 \\
\hline AngKulturang Pilipino Ng MgaWikang Filipino & 1006 \\
\hline WalangPanginoon & 607 \\
\hline SandalingRepleksyon & 550 \\
\hline Ang Sex Education Ni InayUkol Sa Origin Ng Mga Bata & 548 \\
\hline AngAlibughangAnak & 436 \\
\hline AngMangmang at angPari & 317 \\
\hline $\begin{array}{l}\text { AngMgaKagila-gilalas Na Pakikipagsapalaran Ni Juan } \\
\text { Dela Cruz }\end{array}$ & 248 \\
\hline Tinawid Ni PilandokAngIlog & 227 \\
\hline AngAlamatngSaging & 222 \\
\hline Haring Ibon & 135 \\
\hline Kabataan Ng LahingKayumanggi & 101 \\
\hline AngPilipina Sa BagongMilenyo & 94 \\
\hline Kapalaran & 68 \\
\hline Total & $\mathbf{9 7 6 8}$ \\
\hline
\end{tabular}

These words are screened for unique words for each article. The extracted list of unique words was manually transcribed phonetically based on the UP Diksyonaryong Filipino, a monolingual dictionary maintained by the University of the Philippines Center for Languages [13]. Phonemes such as /p:/ /b:/ /m:/ /t:/ /d:/ /n:/ /s:/ /l:/ /k:/ /g:/ were included to denote a longer duration of phoneme pronunciations as compared to its shorter counterparts. The diphthongs /iw/ /ay/ /aw/ /oy/ /ey/ /uy/ were also included as part of the vowel phoneme list.The 2938 unique words are tabulated into an excel spreadsheet wherein the frequency of words, phonetic structure, syllabications, and number of syllables were included from the 9768 word included in the articles.

Table 4: Frequency of Syllable Counts from the extracted unique words

\begin{tabular}{|c|c|c|c|}
\hline Syllable Count & Frequency & $\begin{array}{c}\text { Syllable } \\
\text { Count }\end{array}$ & Frequency \\
\hline 1-syllable & 101 & 7-syllable & 23 \\
\hline 2-syllable & 780 & 8-syllable & 7 \\
\hline 3-syllable & 912 & 9-syllable & 2 \\
\hline 4-syllable & 740 & 10-syllable & 1 \\
\hline 5-syllable & 299 & 13-syllable & 1 \\
\hline 6-syllable & 72 & Total & $\mathbf{2 9 3 8}$ \\
\hline
\end{tabular}


International Journal of Advanced Research in Computer and Communication Engineering

Vol. 4, Issue 1, January 2015

A tabulation of the frequency of number of syllabications were extracted from the excel spreadsheet as a basis of selecting the best syllabically homogeneous words from the 2938 unique words. Table 5: 2-syllable words and 3-syllablewords and occurrences.

\begin{tabular}{|c|c|c|c|}
\hline Syllable Count & 1 occurrence & >1 occurrences & Total \\
\hline 2-syllable & 457 & 323 & 780 \\
\hline 3-syllable & 663 & 249 & 912 \\
\hline
\end{tabular}

From the 780 and 9122 -syllable and 3-syllable words found in the list, a total of 3232 -syllable and 2493 syllable words were extracted of which have more than 1 frequency of occurrence in the list to ensure commonality of words. These words are grouped according to their phonetic structure, constituting at least $80 \%$ of the total numbers of the 3-syllable and 2syllable words.

Table 6:Phonetic Structure of 2-Syllable unique word list

\begin{tabular}{|l|l|l|l|}
\hline $\begin{array}{c}\text { Phonetic } \\
\text { Structure }\end{array}$ & Frequency & Vowel & Consonants \\
\hline cv-cvc & 130 & 260 & 390 \\
\hline cv-cv & 61 & 122 & 122 \\
\hline v-cvc & 43 & 86 & 86 \\
\hline cvc-cv & 27 & 54 & 81 \\
\hline cvc-cvc & 25 & 50 & 100 \\
\hline cv-vc & 13 & 26 & 26 \\
\hline v-vc & 12 & 24 & 24 \\
\hline vc-cvc & 3 & 6 & 9 \\
\hline ccv-cv & 3 & 6 & 9 \\
\hline vc-cv & 2 & 4 & 4 \\
\hline cv-vc & 1 & 1 & 2 \\
\hline v-v & 1 & 2 & 0 \\
\hline cvc-ccv & 1 & 2 & 4 \\
\hline cvc-ccvc & 1 & 2 & 5 \\
\hline cv-v & 1 & 2 & 1 \\
\hline Total & $\mathbf{3 2 3}$ & $\mathbf{6 4 6}$ & $\mathbf{8 4 9}$ \\
\hline
\end{tabular}

Table 7:Phonetic Structure of 3-Syllable unique word list

\begin{tabular}{|l|l|l|l|}
\hline $\begin{array}{l}\text { Phonetic } \\
\text { Structure }\end{array}$ & Frequency & Vowel & Consonant \\
\hline cv-cv-cvc & 100 & 300 & 400 \\
\hline cv-cv-cv & 38 & 114 & 114 \\
\hline cv-cvc-cvc & 15 & 45 & 75 \\
\hline cvc-cv-cvc & 14 & 42 & 70 \\
\hline v-cv-cvc & 11 & 33 & 33 \\
\hline cvc-cv-cv & 11 & 33 & 44 \\
\hline cv-cv-vc & 9 & 27 & 27 \\
\hline v-cv-cv & 8 & 24 & 16 \\
\hline cv-cvc-cv & 8 & 24 & 32 \\
\hline cv-v-cvc & 6 & 18 & 18 \\
\hline cv-cv-v & 3 & 9 & 6 \\
\hline cv-vc-cvc & 3 & 9 & 12 \\
\hline
\end{tabular}

\begin{tabular}{|l|l|l|l|}
\hline cv-v-cv & 3 & 9 & 6 \\
\hline cvc-cvc-cv & 3 & 9 & 15 \\
\hline cvc-cvc-cvc & 2 & 6 & 12 \\
\hline cvc-cv-vc & 2 & 6 & 8 \\
\hline vc-ccv-cvc & 1 & 3 & 5 \\
\hline vc-cvc-cvc & 1 & 3 & 5 \\
\hline vc-cv-cvc & 1 & 3 & 4 \\
\hline vc-cv-ccvc & 1 & 3 & 5 \\
\hline v-v-cvc & 1 & 3 & 2 \\
\hline v-cvc-cvc & 1 & 3 & 4 \\
\hline cvc-v-cv & 1 & 3 & 3 \\
\hline cvc-vc-cvc & 1 & 3 & 5 \\
\hline cvc-cv-v & 1 & 3 & 3 \\
\hline cv-cv-ccv & 1 & 3 & 4 \\
\hline ccvc-cv-cv & 1 & 3 & 5 \\
\hline ccv-cvc-cv & 1 & 3 & 5 \\
\hline ccv-cv-cv & 1 & 3 & 4 \\
\hline Total & $\mathbf{2 4 9}$ & $\mathbf{7 4 7}$ & $\mathbf{9 4 2}$ \\
\hline
\end{tabular}

214 of the 3-syllable words are represented with the following phonetic structures: $C V-C V-C V C, C V-C V-C V, C V$ -

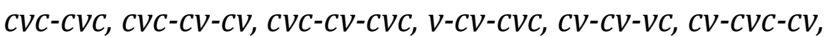
and $v-c v-c v$ with the frequencies of $0.416,0.1526$, $0.0602,0.0441,0.0562,0.0441,0.0361,0.0321$, and 0.0321 respectively. While 261 of the 2 -syllable words are represented with the following phonetic structures: $c v-c v c, C v-c v, v-c v c$, and $c v c-c v$ with the frequencies of $0.4024,0.1889,0.1331$, and 0.0836respectively.A frequency of each phoneme is calculated with the formula:

Where:

$$
F=\frac{\sum\left(f_{p w} \cdot f_{w}\right)}{n}
$$

$$
\begin{array}{ll}
\mathrm{F} & \begin{array}{l}
\text { frequency of phonemes represented in } \\
\text { the word list }
\end{array} \\
f_{p w} & \text { frequency of phoneme in a word } \\
f_{w} & \text { frequency of word occurrence } \\
\mathrm{n} & \text { total number of phonemes in the word } \\
& \text { list }
\end{array}
$$

This value is compared to the acceptance value (threshold value) with the formula:

Where:

$$
\mathrm{aV}=\frac{1}{(x \cdot m)}
$$

$\mathrm{aV}$ acceptance value/threshold value

$\mathrm{x}$ average of the vowels/consonants in a phonetic structure

$\mathrm{m}$ total number of words

Table 8: Acceptance values of phonemes for the 2-syllable and 3-syllable words

\begin{tabular}{|l|c|c|}
\hline & Vowel & Consonants \\
\hline 2-syllable words & 0.0023 & 0.0018 \\
\hline 3-syllable words & 0.0015 & 0.0012 \\
\hline
\end{tabular}

The acceptance value for the frequency of vowels from the 2-syllable word list is 0.0023 and 0.0018 for the consonants based on the 2612 -syllable words while the 
acceptance value for the vowels from the 3 -syllable word respondents ( 25 male, and 25 female). All members of the list is 0.0015 and 0.0012 for consonants based on 214 group have Filipino as their primary and native language, words in list. These values are compared from the with no speaking ailments, and were at their proper frequency of each phoneme in the list to validate if the dispositions.

phoneme is well represented. Phonemes lower than the acceptance values were not represented, thus the words including the phonemes were removed from the accumulated list while frequencies higher or equal to the acceptance values were well represented by the list.

From the 20 basic phonemes of the Filipino language, 16 phonemes were added (10 long consonants, and 6 diphthongs). 5 phonemes were not represented in the 2syllable word list (/m:/, /g:/, /iw/, /ey/, /uy/) since the frequencies of these phonemes are less than the acceptable value of 0.0023 for vowels, and 0.0018 for consonants. 4 phonemes were not represented in the 3-syllable word list (/m:/, /g:/, /iw/, /ey/, /uy/) which are less than the acceptable values of 0.0015 for vowels and 0.0012 for consonants. The total number of words represented by the 3 -syllable word list is 214 , and 261 for the 2-syllable word list. Thus, the final list for the phonetically balanced word list for the Filipino Language will be 257 for 2-syllable words, and 212 for 3 -syllable words.

Table 9: Word count for the 3-syllable and 2-syllable list with the number of represented phonemes

\begin{tabular}{|c|c|c|c|c|c|}
\hline & $\begin{array}{l}\text { Phoneme } \\
\text { Structure }\end{array}$ & $\begin{array}{c}\text { Freq } \\
\text { uenc } \\
y \\
\end{array}$ & $\begin{array}{c}\text { Vow } \\
\text { el }\end{array}$ & $\begin{array}{c}\text { Cons } \\
\text { onan } \\
\mathbf{t}\end{array}$ & $\begin{array}{c}\text { Phoneme } \\
\text { No }\end{array}$ \\
\hline \multirow{5}{*}{ PBW2 } & cv-cve & 127 & 254 & 381 & \multirow{5}{*}{31} \\
\hline & $\mathrm{cV}-\mathrm{cv}$ & 61 & 122 & 122 & \\
\hline & $\mathrm{v}-\mathrm{cvc}$ & 43 & 86 & 86 & \\
\hline & cve-cv & 26 & 52 & 78 & \\
\hline & total & 257 & 514 & 667 & \\
\hline \multirow{10}{*}{ PBW3 } & cv-cv-cve & 103 & 309 & 412 & \multirow{10}{*}{32} \\
\hline & $\mathrm{cV}-\mathrm{cV}-\mathrm{cV}$ & 41 & 123 & 123 & \\
\hline & cV-cve-cve & 15 & 45 & 75 & \\
\hline & cve-cv-cve & 11 & 33 & 55 & \\
\hline & $\mathrm{V}-\mathrm{cV}-\mathrm{cvc}$ & 10 & 30 & 30 & \\
\hline & $\mathrm{cvc}-\mathrm{cv}-\mathrm{cv}$ & 9 & 27 & 36 & \\
\hline & $C V-C V-V C$ & 7 & 21 & 21 & \\
\hline & $\mathrm{V}-\mathrm{CV}-\mathrm{CV}$ & 8 & 24 & 16 & \\
\hline & $\begin{array}{c}\mathrm{CV}-\mathrm{CVC}- \\
\mathrm{CV}\end{array}$ & 8 & 24 & 32 & \\
\hline & total & 212 & 636 & 800 & \\
\hline
\end{tabular}

\section{B. Phonetically Balanced Speech Corpus}

The speech corpus is a requirement for the development of automatic speech recognition systems. The speech corpora used in this study were gathered from the 257 2-syllable word list (PBW2) and 212 3-syllable word list (PBW3). These words were recorded for the training and testing of words using an ASR system developed with HMM. The recorded speech data were done by 50 fluent Filipino

\section{TEST OF PHONETICALLY BALANCED SPEECH}

Hidden Markov Models (HMM) are probabilistic models [12] used for modeling stochastic sequence with underlying finite state structure. Speech Corpus for PBW2 and PBW3was prepared for training and the design decisions concerning the models are taken. HTK toolkit was used to develop the speech training and the utterances were encoded to Mel-frequency cepstral coefficient (MFCC) vectors. Data Preparation steps are shown in Figure 1.

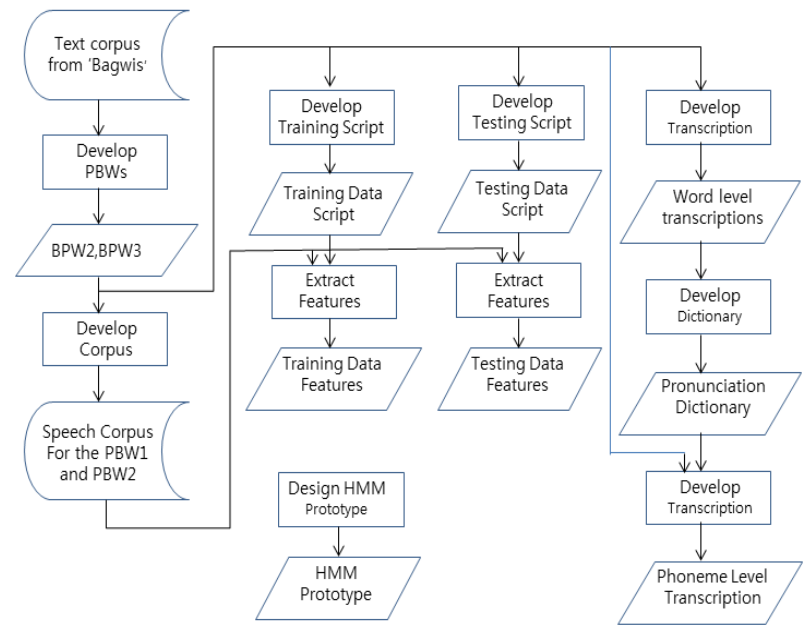

Figure 1:Diagram showing the steps in Data Preparation

From the text corpus, two PBW lists(PBW2 and PBW3) were developed. These PBW lists were used to create a recorded data(speech corpus) and a word level transcription. Phoneme level transcriptions was created from the word level transcriptions by using a pronunciationdictionary that gives each word the sequence of phones that make up the pronunciation ofthisword. These PBW lists were used as a training data. This was tested through dependent and independent speaker test to determine the recognition accuracy on word level and phoneme level recognition.

\section{A. Speech Data(corpus)}

The recordings were done in an isolated room using a unidirectional microphone connected to a computer with input speech sampled at $16 \mathrm{kHz}$ at mono using a linear PCM and saved as waveform file format (*wav). A distance of approximately 5-10 centimeters is used between the mouth of the speakers and the microphone used. The speakers were asked to utter the words from the PBW2 and PBW3 word lists clearly. The speeches collected were used as training data, and test data. The training data were recorded by 25 female and 25 male speakers with 2 sets of word utterances. The test data were grouped as 'speaker dependent' and 'speaker independent'. 
The speaker dependent speech were taken from the same speakers from the training data ( 25 female and 25 male), which recorded another set of word utterances while the speaker independent speech were taken from 5 female and 5 male speakers not included in the training data which recorded a set of word utterances

\section{B. Feature Specifications}

HTK tool HCopywas used to translate audio files to feature vector files in the type of Mel-Frequency Cepstral Coefficients (MFCC) according to the file of script. The main feature extraction parameters used in the studyconsists of 39 dimensional feature vectors of 13 MFCCs, the first and second derivatives. The window size is set to $25 \mathrm{~ms}$ with ashift rate of $10 \mathrm{~ms}$ using a hamming windowing. The pre-emphasis coefficient value is set to 0.97 .

\section{HMM Acoustic Model Specifications}

The speech data are trained into a 7-stateHMM for the PBW2, and 9-state HMM for PBW3 for word level while for the phoneme level, a 5-state HMM was used both for PBW2 and PBW3with the first and the last state characterized as the non-emitting entry and exit states representing null in the speech data. Observation probability of each state consists oneGMM(Gaussian Mixture Model) for all cases. The training is performed through multiple iterations to re-estimate the parameters using the Baum-Welch re-estimation algorithm.In this study, a total of 20 re-estimation iterations were conducted. A set of 31 HMMs is generated for PBW2 and a set of 32 HMMs for PBW3.

\section{RESULTS}

In order to estimate the efficiency of the phonetically balanced words list developed above, two sets of HMM created based on the two PBW lists were tested against two types of speakers: one which is involved in the training (dependent speakers) and the other which is only involved in the testing (independent speakers).Table 10 shows the word-level recognition rate achieved in the [14]. As shown in Table 11 the performance evaluation of phoneme-level recognition was conducted in 1181phonemes of 257 words and 1436 phonemes of 212 words forPBW2 and PBW3 respectively. In a 2-syllable word list (PBW2), an average accuracy rate of $88.95 \%$ for speaker dependent and $82.57 \%$ for speaker independent test were achieved. For 3-syllable word list (PBW3), the recognizer achieved an accuracy rate of $90.28 \%$ for speaker dependent and $83.30 \%$ for speaker independent test.

Table 10:Performance Evaluation Results for word level

$$
\text { recognition }
$$

\begin{tabular}{|c|c|c|}
\hline & $\begin{array}{c}\text { Dependent } \\
\text { Speaker }\end{array}$ & Independent Speaker \\
\hline PBW2 & $93.25 \%$ & $88.67 \%$ \\
\hline PBW3 & $99.53 \%$ & $96.30 \%$ \\
\hline
\end{tabular}

Table 11:Performance Evaluation Results for phoneme level recognition for31 phonemes in 257 words(1181 phonemes) in PAW2 and 32 phonemes in 212 words(1436 phonemes) in PBW3

\begin{tabular}{|c|c|c|c|c|c|c|}
\hline & & \multicolumn{4}{|c|}{ Testing Data } & \multirow{2}{*}{$\begin{array}{c}\text { accurac } \\
\mathbf{y}\end{array}$} \\
\hline & & $\begin{array}{c}\text { phone } \\
\text { mes }\end{array}$ & $\begin{array}{l}\text { spea } \\
\text { kers }\end{array}$ & sets & total & \\
\hline \multirow{2}{*}{ PBW2 } & DPD* & \multirow{2}{*}{1,181} & 45 & 2 & 106,290 & $88.95 \%$ \\
\hline & IND* & & 5 & 2 & 11,810 & $82.57 \%$ \\
\hline \multirow{2}{*}{ PBW3 } & DPD* & \multirow{2}{*}{1,436} & 45 & 2 & 129,240 & $90.28 \%$ \\
\hline & IND* & & 5 & 2 & 14,360 & $83.30 \%$ \\
\hline
\end{tabular}

DP*: dependent, IND*:independent

\section{CONCLUSION}

In this paper, we developed two sets of phonetically balanced word (PBW) lists in Filipino and two sets of phoneme-level HMMs(Hidden Markov Model). Two PBW lists were created based on textbooks used in public school in Philippines and used to develop speech corpus with the fifty speakers of 25 males and 25 females.In a 2syllable word list (PBW2) with 257 words and 31 phonemes, $31 \mathrm{HMMs}$ were trained and an average accuracy rate of $88.95 \%$ for speaker dependent and $82.57 \%$ for speaker independent test were achieved. For 3-syllable word list (PBW3) with in 212 words and 32 phonemes, 32 HMMs were trained and the recognizer achieved an accuracy rate of $90.28 \%$ for speaker dependent and $83.30 \%$ for speaker independent test.The developed PBW lists can be improved by increasing the size of text corpora which will also increase the efficiency of the phoneme distribution in the words of the PBW list

\section{ACKNOWLEDGEMENT}

This research was supported by the research fund of Hanbat National University in 2011.

\section{REFERENCES}

[1] Navarro, R. D., "Recognition of Tagalog Alphabets Using the Hidden Markov Model" in $10^{\text {th }}$ National Convention in Statistics (2007).

[2] R. C. L. Guevara, M. Co, E. Tan, I. D. Garcia, E. Espina, R. Ensomo, and R. Sagum, "Development of a Filipino Speech Corpus" in Proc. 3rd National ECE Conference, (2002).

[3] Rabiner, L. R. and Juang, B. H., "Fundamentals of Speech Recognition", Englewood Cliffs, NJ, Prentice Hall, (1993).

[4] Kumar, K., "Hindi Speech Recognition System using HTK", International Journal of Computing and Business Research (Vol 2, Issue 2), (2011).

[5] Sakti S., Isotani, R., Kawai H., and Nakamura, S.,"The Use of Indonesian Speech Corpora for Developing a Filipino Continuous Speech Recognition System", (2010) http://wika.pbworks.com/w/page/8021671/Kasaysayan, "EbolusyongngAlpabetong Filipino", (Retrieved 2012).

[7] Lewis, P. M., "Languages of Philippines. Ethnologue:Languages of the World" (16th ed.), (2009).

[8] Santiago, A., Tiangco, N., "MakabagongBalarilang Pilipino", (1985).

[9] Ferrer O., "Speech audiometry: a discrimination test for Spanish language",(1960).

[10] Rosas y De Mendizabal B.,"Speech Audiometry in English, Portuguese and Spanish", (1958).

[11] Travero, K., "UP Diksyonaryong Filipino", 1st Edition, UP Publishing Press, Quezon City, (2001). 
[12] Hachkar, Z., Farchi A., Mounir. B., El Abbadi J., "Building HMM recognizer for Arabic Digits using HTK", (2007)

[13] Fajardo A., Kim Y.J., "Test of Vowels in Speech Recognition using Continuous Hidden Markov Model and Development of Phonetically Balanced-Words in the Filipino Language", $5^{\text {th }}$ International Conference for Engineering and Business Education$\operatorname{ICEBE}(2012)$

[14] Fajardo A., Kim Y.J., "Development of Filipino Phonetically Balanced-Words and Test using Hidden Markov Model", International Conference for Artificial Intelligence-ICAI (2013)

\section{BIOGRAPHIES}

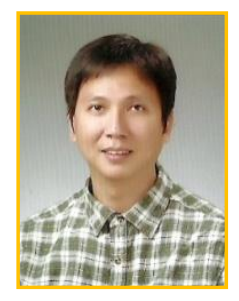

Arnel C. Fajardo received the B.S. degree in electrical engineering in 1991 from Mapua Institute of Technology,the M.S. degree in computer science in 1999 from La Salle University both in Manila, and the Ph.D. degree in computer engineering from Hanbat National University, Daejon, South Korea in 2014.

Heis a Senior Lecturer at La Consolacion College Manila, Philippines and a program evaluator/accreditorof information technology and computer science of PAASCU (Philippine Accrediting Association of Schools, Colleges and Universities). He is an author of more than 10 articles in journals and conference proceedings. His research interest includes speech recognition, Artificial intelligence and engineering education.

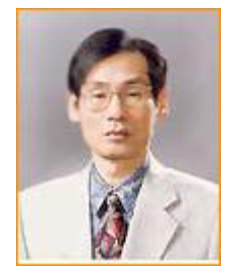

Yoon-joong Kim received the B.S. degree in electronics engineering in 1981, the M.S. degreein computer engineering in 1983 and the Ph.D.degree in computer engineering in 1999 from Chungnam National University, Daejeon, South Korea. He is a member of recognized scientific institutions in Korea such as The Korean Institute of Information Scientists and Engineers, Korea Multimedia Society, The Acoustical Society of Korea, The Institute of Electronics and Information Engineers and Korean Society of Cognitive Science. Since 1984, he has been a full professor of Hanbat National University of the Department of Computer Engineering. $\mathrm{He}$ has conducted more than 20 government related projects in his field of expertise. He is an author of more than 30 articles in journals and conference proceedings. His research interest includes Artificial Intelligence, Digital Signal Processing, Speech Recognition, Web Technology and Service Oriented Architecture. 